\title{
Candidemia em um hospital terciário brasileiro: incidência, frequiência das diferentes espécies, fatores de risco e suscetibilidade aos antifúngicos
}

\author{
Candidemia in a Brazilian tertiary care hospital: incidence, frequency \\ of different species, risk factors and antifungal susceptibility
}

\author{
João Cesar Beenke França ${ }^{1}$, Clea Elisa Lopes Ribeiro ${ }^{1}$ e Flávio de Queiroz-Telles ${ }^{1}$
}

\begin{abstract}
RESUMO
São apresentados os resultados de estudo transversal e observacional sobre candidemia realizado no Hospital de Clínicas da Universidade Federal do Paraná. No período de janeiro de 2001 a dezembro de 2004, foram analisados 100 episódios de candidemia. A incidência foi de 1,27 por 1.000 internações, sendo Candida spp o oitavo agente mais isolado nas infecções da corrente sanguínea. A idade variou de 5 dias a 89 anos com uma média de 32 anos, $60 \%$ dos casos ocorreram em adultos (66\% > 50 anos) e 40\% em crianças $(52 \%<1$ ano). Cinqüenta e nove pacientes estavam internados em enfermarias e $41 \mathrm{em}$ unidade de terapia intensiva. Candida albicans foi a espécie mais (59\%) freqüente, seguida por Candida tropicalis (15\%), Candida parapsilosis (9\%). As condições associadas mais (97\%) freqüentes foram uso de antibióticos, cateter venoso central (77\%), bloqueador $\mathrm{H}_{2}(57 \%)$, nutrição parenteral total (49\%) internamento em unidade de terapia intensiva (41\%). Dos 51 isolados testados, 3 de Candida glabrata apresentaram suscetibilidade dose-dependente ao fluconazol e eram resistentes ao itraconazol. Uma amostra de Candida kruse $i$ apresentou suscetibilidade dose-dependente ao fluconazol, e uma de Candida pelliculosa suscetibilidade dose-dependente ao itraconazol. Na população de estudo, $68 \%$ receberam tratamento antifúngico, no entanto a mortalidade foi de $56 \%$.
\end{abstract}

Palavras-chaves: Candidemia. Candida. Sepse. Azólicos.

\begin{abstract}
Results from a cross-sectional observational study on candidemia conducted at Hospital de Clínicas, Federal University of Paraná, are presented. From January 2001 to December 2004, one hundred candidemia cases were evaluated. The incidence was 1.27 episodes per 1,000 hospitalizations and Candida was the eighth most frequently isolated agent from bloodstream infections. The patients' ages ranged from five days to 89 years, with a mean of 32 years. Sixty percent of the cases occurred in adults $(66 \%>50$ years old) and $40 \%$ in children $(52 \%<$ one year old). Fifty-nine percent had been admitted to medical wards and $41 \%$ to the intensive care unit. Candida albicans was the most (59\%) frequent species followed by Candida tropicalis (15\%) and Candida parapsilosis (9\%). The most (97\%) prevalent coexisting conditions were the use of antibacterial drugs, central venous catheter (77\%), $\mathrm{H}_{2}$ blockers (57\%), total parenteral nutrition (49\%) and admission to the intensive care unit (41\%). Out of the 51 isolates tested, three of Candida glabrata presented dose-dependent susceptibility to fluconazole and resistance to itraconazole. One sample of Candida kruse $i$ presented dose-dependent susceptibility to fluconazole and one of Candida pelliculosa presented dose-dependent susceptibility to itraconazole. Among the study population, $68 \%$ received antifungal therapy, but the mortality was $56 \%$.
\end{abstract}

Key-words: Candidemia. Candida. Sepsis. Azoles.

Sepse é a principal causa de óbito entre os pacientes internados e gravemente doentes, sendo que um aumento de $164.000(82,7 / 100.000$ pacientes $)$ para 660.000 (240/100.000 pacientes) casos anuais desta complicação foi observada nos EUA num período de 22 anos (1979-2000). A freqüência de infecção da corrente sangüínea causada por fungos aumentou $207 \%$ neste mesmo período ${ }^{21}$. Destas infecções, $80 \%$ são causadas por espécies de leveduras do gênero Candida ${ }^{17}$, sendo considerada a quarta causa de sepse $^{12} 25$, correspondendo a 5\%-10\% segundo dados do Nosocomial Infection Sueveillance System ${ }^{13}$. Candida albicans é o agente mais prevalente $2^{2} 10112631$. No entanto, a freqüência de espécies não albicans vem aumentando ${ }^{10}$, principalmente pelo uso prévio de fluconazol ${ }^{122} 31$.

1. Serviço de Infectologia, Hospital de Clínicas, Universidade Federal do Paraná, Curitiba, PR.

Endereço para correspondência: Dr. João Cesar Beenke França. R. São Domingos 873, Sobrado 2, Pilarzinho, 82100-390 Curitiba, PR.

Tel: $55413338-1835$

e-mail: jofrancb@brturbo.com.br

Recebido para publicação em: 26/09/2006

Aceito em: 17/12/2007 
As manifestações clínicas são inespecíficas, sendo a febre o sinal mais comum ${ }^{243031}$. A origem endógena da candidemia, por fenômeno de translocação, é a principal, entretanto algumas espécies (Candida parapsilosis) podem ter origem exógena, principalmente pelo uso do cateter venoso central, soluções glicosadas e pelas mãos colonizadas dos profissionais que prestam atendimento ao paciente. 0 diagnóstico é um desafio, pois o principal exame para diagnóstico, a hemocultura, só é positiva em aproximadamente $50 \%$ dos $\operatorname{cas}^{12620}$, e a mortalidade é alta, estimada entre $40 \%$ e $60 \%$ com mortalidade atribuível de $38 \%^{281014232631}$. Os principais objetivos do presente estudo foram avaliar, em hospital público, a incidência das candidemias, as espécies prevalentes de Candida, fatores de risco e a suscetibilidade aos antifúngicos.

\section{MATERIAL E MÉTODOS}

Delineamento do estudo. Este é um estudo transversal, descritivo, observacional, individual, contemporâneo e histórico.

População e período de estudo. Foram estudados os casos de candidemia ocorridos no Hospital de Clínicas da Universidade Federal do Paraná no período de janeiro de 2001 a dezembro de 2004.

A instituição. O Hospital de Clínicas da Universidade Federal do Paraná (HC/UFPR) é um hospital terciário e está situado na Cidade de Curitiba, no Estado do Paraná, região Sul do Brasil, com capacidade para 635 leitos, incluindo a maioria das especialidades da clínica médica e cirúrgica, serviço de transplante de medula óssea, transplante hepático, unidade de terapia intensiva (UTI) de adulto (clínica e cirúrgica), UTI neonatal, UTI pediátrica, serviço de pronto atendimento e emergência de adultos e pediátrico, quimioterapia de alto risco, infectologia de adulto e pediátrica, ginecologia e obstetrícia.

Critérios de inclusão. Foram incluídos pacientes com pelo menos uma hemocultura positiva para Candida spp e com sinais e sintomas clínicos de infecção.

Critérios de exclusão. Hemoculturas positivas para Candida spp com ausência de sinais e sintomas clínicos de infecção.

Dados coletados. Os dados coletados foram baseados em uma ficha clínica padrão. Registraram-se dados demográficos, como idade (sendo consideradas crianças pacientes com idade $\leq 14$ anos, pois este é o limite utilizado pelo Hospital de Clínicas da UFPR para fins de internação, segundo critério do Sistema Único de Saúde), gênero e local de internação no momento da candidemia, condições clínicas de base, as espécies de Candida e os seguintes condições associadas: hospitalização prévia, colonização, ventilação mecânica, nutrição parenteral total, diálise, neutropenia, presença de cateter venoso central, tratamento com antibióticos, tratamento com corticosteróide, quimioterapia, imunossupressão, medicamentos anti-retrovirais, bloqueador $\mathrm{H}_{2}$, antifúngico prévio, internamento na UTI e cirurgia abdominal. Foi ainda registrado se o paciente recebeu tratamento antifúngico e sua evolução (alta ou óbito).
Definições. Episódio de candidemia: quando pelo menos uma hemocultura é positiva para Candida spp em presença de sinais e sintomas de infecção (febre, hipotensão, leucocitose) dentro de um período de 30 dias. Se outra hemocultura positiva para Candida spp ocorre durante esses 30 dias, pela mesma espécie ou por uma espécie diferente, esta(s) hemocultura(s) é (são) registrada(s) como sendo o mesmo episódio. Se o paciente apresentava outra hemocultura positiva para Candida spp em período maior que 30 dias em relação à hemocultura inicial, este era considerado um novo episódio.

Candidemia inicial ou incidente: considerado como tal o primeiro episódio de candidemia.

Condições clínicas de base: a patologia que motivou a internação, as intercorrências, e as patologias preexistentes que estavam ativas nos três meses antes da candidemia inicial.

Condições associadas: a colonização foi considerada fator de risco quando ocorreu nos 14 dias precedentes à candidemia inicial. Ventilação mecânica, nutrição parenteral total e presença de cateter venoso central foram consideradas como fator de risco se estavam presentes pelo menos nas 24 horas prévias à candidemia inicial. A neutropenia e a diálise foram consideradas se estavam presentes pelo menos 72 horas antes da candidemia inicial. Em relação às medicações, estas foram consideradas como fator de risco se estavam sendo utilizadas nos 14 dias precedentes à candidemia inicial.

Drogas imunossupressoras foram consideradas: metotrexate, hidroxicloroquina, ciclofosfamida, micofenolato, mofetil, etanercet e infliximab. Neutropenia foi definida para número de neutrófilos menores que $500 / \mathrm{mm}^{3}$, neutropenia severa quando o número de neutrófilos estava menor que $100 / \mathrm{mm}^{3}$, e neutropenia prolongada quando a sua duração era maior que 10 dias. Febre: temperatura axilar $\geq 37,8^{\circ} \mathrm{C}$. Hipotensão: pressão sistólica $<90 \mathrm{mmHg}$. Pacientes foram considerados crianças com idade $\leq 14$ anos.

Tratamento: se algum tratamento antifúngico foi instituído até 30 dias da candidemia inicial.

Evolução: se o paciente recebeu alta após a recuperação da infecção ou evoluiu para óbito.

Procedimentos microbiológicos. As hemoculturas positivas foram detectadas pelo sistema automatizado Bact/Alert; Organon Teknica, USA e a identificação pelo sistema automatizado WalkAway até 2002, e posteriormente a este ano pelo bioMérieux Vitek, France, sendo realizados conjuntamente procedimentos clássicos como auxanograma e tubo germinativo.

Os isolados de 2001-2002 não foram submetidos aos testes de suscetibilidade, pois várias amostras não se encontravam mais viáveis.

Os isolados de Candida spp do período 2003-2004 foram enviados ao Laboratório Especial de Micologia da UNIFESP, São Paulo para a realização de testes de suscetibilidade, e a confirmação da identificação. A suscetibilidade foi realizada pelo método de microdiluição em caldo, conforme documento M27-A da National Committee for Clinical Laboratory Standars (NCCLS).

Análise estatística. Incidência foi calculada pelo número de candidemias por 1.000 internações. Para análise estatística, 
foi utilizado o programa Microsoft Excel da Microsoft e o JPM 5.1 da SAS Institute. Variáveis categóricas foram analisadas através do teste Qui-quadrado e teste exato de Fisher. Variáveis contínuas foram analisadas utilizado teste de Wilcoxon. Para análise entre as espécies e local de internação, teste de Person. 0 valor de $\mathrm{P} \leq$ que 0,05 foi considerado significativo.

\section{RESULTADOS}

Um total de 100 episódios de candidemia ocorreu no período de 4 anos (2001-2004) com predominância dos adultos e gênero masculino (60\%). A infecção foi mais frequiente em adultos acima de 50 anos (66,6\%). Em 40 casos observados na população pediátrica, $80 \%$ das crianças tinham idade até 4 anos. Entre as doenças de base predominaram as cirurgias do aparelho digestivo, e a maioria dos pacientes estava internada em enfermarias. Os dados demográficos dos pacientes com candidemia são mostrados na Tabela 1 .

A Tabela 2 mostra a distribuição das várias espécies identificadas. Candida albicans ocorreu em 59\% dos episódios seguida por Candida tropicalis (15\%), Candida parapsilosis (9\%), Candida glabrata (7\%). Observa-se também uma predominância de Candida parapsilosis entre as crianças e de Candida glabrata entre os adultos, similar a outros estudos.

Tabela 1 - Dados demográficos dos 40 pacientes no Hospital de Clínicas da Universidade Federal do Paraná, 2001-2004.

\begin{tabular}{|c|c|c|}
\hline Variáveis & Número & Porcentagem \\
\hline \multicolumn{3}{|l|}{ Idade (anos) } \\
\hline$<1$ & 21 & 21,0 \\
\hline $1-4$ & 11 & 11,0 \\
\hline $5-9$ & 4 & 4,0 \\
\hline $10-14$ & 4 & 4,0 \\
\hline $15-19$ & 0 & 0,0 \\
\hline $20-29$ & 9 & 9,0 \\
\hline $30-39$ & 8 & 8,0 \\
\hline $40-49$ & 6 & 6,0 \\
\hline $50-59$ & 15 & 15,0 \\
\hline $60-64$ & 4 & 4,0 \\
\hline$>65$ & 18 & 18,0 \\
\hline \multicolumn{3}{|l|}{ Sexo } \\
\hline masculino & 60 & 60,0 \\
\hline feminino & 40 & 40,0 \\
\hline \multicolumn{3}{|l|}{ Grupo etário } \\
\hline adulto & 60 & 60,0 \\
\hline criança $(\leq 14$ anos $)$ & 40 & 40,0 \\
\hline \multicolumn{3}{|l|}{ Local de internação } \\
\hline enfermaria & 59 & 59,0 \\
\hline UTI & 41 & 41,0 \\
\hline \multicolumn{3}{|l|}{ Doença de base } \\
\hline doença que requereu cirurgia & 43 & 43,0 \\
\hline neoplasia & 25 & 25,0 \\
\hline doença pulmonar & 19 & 19,0 \\
\hline outras & 13 & 13,0 \\
\hline
\end{tabular}

Tabela 2 - Distribuição das espécies de Candida segundo a faixa etária no Hospital de Clínicas da Universidade Federal do Paraná 2001-2004.

\begin{tabular}{|c|c|c|c|c|c|c|}
\hline & \multicolumn{2}{|c|}{ Adultos } & \multicolumn{2}{|c|}{ Crianças } & \multicolumn{2}{|c|}{ Total } \\
\hline & $\mathrm{n}^{\underline{0}}$ & $\%$ & $\mathrm{n}^{\underline{0}}$ & $\%$ & $\mathrm{n}^{\mathrm{0}}$ & $\%$ \\
\hline Candida albicans & 33 & 55,0 & 26 & 65 & 59 & 59,0 \\
\hline Candida tropicalis & 12 & 20,0 & 3 & 7,5 & 15 & 15,0 \\
\hline Candida parapsilosis & 3 & 5,0 & 6 & 15,0 & 9 & 9,0 \\
\hline Candida glabrata & 7 & 11,7 & 0 & 0,0 & 7 & 7,0 \\
\hline Candida spp & 2 & 3,3 & 1 & 2,5 & 3 & 3,0 \\
\hline Candida krusei & 1 & 1,7 & 1 & 2,5 & 2 & 2,0 \\
\hline Candida guilliermondii & 1 & 1,7 & 1 & 2,5 & 2 & 2,0 \\
\hline Candida pelliculosa & 1 & 1,7 & 1 & 2,5 & 2 & 2,0 \\
\hline Candida lusitaniae & 0 & 0,0 & 1 & 2,5 & 1 & 1,0 \\
\hline Total & 60 & 100,0 & 40 & 100,0 & 100 & 100,0 \\
\hline
\end{tabular}

Entre as condições associadas mostradas na Tabela 3, as mais freqüentes foram uso de antibióticos (97\%), presença de cateter venoso central (77\%), bloqueador $\mathrm{H}_{2}(57 \%)$, nutrição parenteral total (49\%), internamento na unidade de terapia intensiva (41\%), uso de corticosteróide (39\%) e cirurgia do aparelho digestivo (35\%). É importante destacar o pequeno (10\%) número de pacientes com neutropenia. Neste estudo, 51 amostras foram analisadas quanto à suscetibilidade: três isolados de Candida glabrata apresentaram suscetibilidade dose-dependente ao fluconazol e resistência ao itraconazol, um isolado de Candida kruse $i$ apresentou suscetibilidade dose-dependente ao fluconazol,

Tabela 3 - Condições associadas nos pacientes com candidemia no Hospital de Clínicas da Universidade Federal do Paraná, 2001-2004

\begin{tabular}{|c|c|c|}
\hline Condições associadas & Número* & Porcentagem \\
\hline Antibióticos & 97 & 97,0 \\
\hline Cateter venoso central & 77 & 77,0 \\
\hline Bloqueador $\mathrm{H}_{2}$ & 57 & 57,0 \\
\hline Nutrição parenteral total & 49 & 49,0 \\
\hline Internação na UTI & 41 & 41,0 \\
\hline Corticosteróide & 39 & 39,0 \\
\hline Cirurgia do aparelho digestivo & 35 & 35,0 \\
\hline Hospitalização prévia & 35 & 35,0 \\
\hline Colonização & 33 & 33,0 \\
\hline Ventilação mecânica & 26 & 26,0 \\
\hline Quimioterapia & 13 & 13,0 \\
\hline Antifúngico prévio & 12 & 12,0 \\
\hline Neutropenia & 10 & 10,0 \\
\hline Diálise & 8 & 8,0 \\
\hline Imunossupressor & 5 & 5,0 \\
\hline Anti-retroviral & 3 & 3,0 \\
\hline
\end{tabular}

*Número de vezes que aparecem.

e um isolado de Candida pelliculosa suscetibilidade dosedependente ao itraconazol (Tabela 4 e 5).

A taxa de mortalidade foi de $56 \%$, apesar da maioria (68\%) ter recebido tratamento antifúngico. No grupo de óbitos predominaram os adultos em relação às crianças $(60 \%$ e $40 \%$, respectivamente) os pacientes internados em UTI em relação aos das enfermarias (73,8\% e 43,1\% respectivamente). Quanto às espécies, houve significância estatística para Candida glabrata, onde seis, de um total de sete estavam no grupo de óbitos. 
Tabela 4 - Suscetibilidade das Candidas aos antifúngicos no Hospital de Clínicas da Universidade Federal do Paraná 2001-2004.

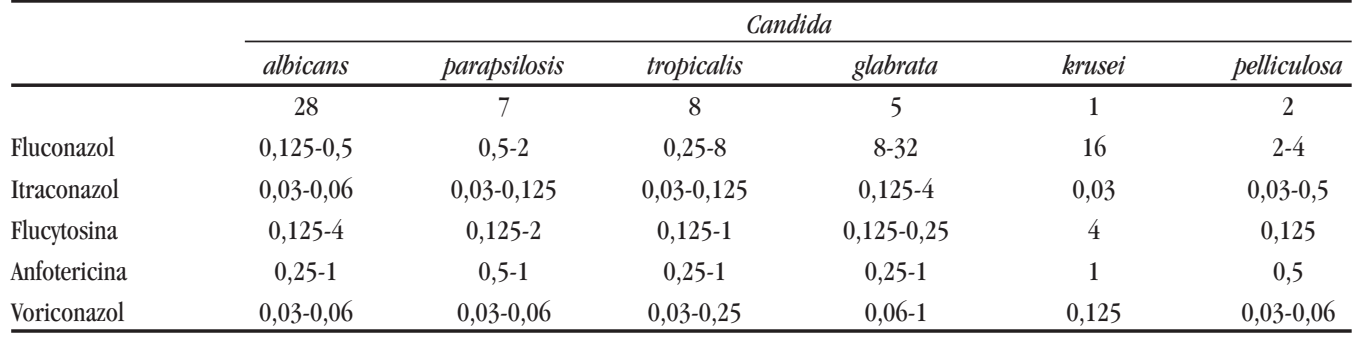

Nota: susceptibilidade em $\mu \mathrm{g} / \mathrm{ml}$.

Tabela 5 - Perfil das Candidas com susceptibilidade reduzida no Hospital de Clínicas da Universidade Federal do Paraná 2001-2004.

\begin{tabular}{lcccccc}
\hline Número & Espécie de Candida & Anfotericina B & Fluconazol & Itraconazol & Flucytosina & Voriconazol \\
\hline 30 & glabrata & 0,5 & 16 & 1 & 0,125 & 1 \\
92 & glabrata & 0,25 & 16 & 1 & 0,125 & 0,25 \\
25 & glabrata & 1 & 32 & 4 & 0,25 & 0,06 \\
48 & pelliculosa & 0,5 & 4 & 0,5 & 0,125 & 0,03 \\
52 & krusei & 1 & 16 & 0,03 & 4 & 0,125 \\
\hline
\end{tabular}

Nota: suscetilidade em $\mu \mathrm{g} / \mathrm{ml}$.

\section{DISCUSSÃo}

A sepse por Candida spp vem aumentando nos últimos anos ${ }^{1}$, o diagnóstico é difícil ${ }^{12} 620$, e a mortalidade é alta ${ }^{28} 10142326$.

A incidência de candidemia observada no estudo (1,27 por 1.000 internações) ficou muito acima dos dados europeus e americanos $^{13}$. A razão desta maior incidência não é conhecida. Porém, destaca-se que o estudo foi realizado em um hospital universitário e público, não se conhecendo a realidade de incidência em hospitais privados brasileiros. Algumas possíveis causas seriam a diferença nos recursos disponíveis nos hospitais públicos, assim como o desconhecimento desta complicação (candidemia) pelo não especialista, não contemplando o uso profilático em pacientes muito vulneráveis, como os internados na unidade de terapia intensiva. A dificuldade de colocar em prática procedimentos consagrados de prevenção da infecção hospitalar, como higienização das mãos, para evitar a transmissão através das mãos colonizadas da equipe que presta atendimento ao paciente, assim como o número insuficiente de profissionais que prestam atendimento ao paciente em áreas críticas, principalmente nas unidade de tratamento intensivo.

As espécies não albicans estão classicamente associadas à neoplasia, neutropenia e o uso prévio de fluconazol entre outros $^{12231}$. A pequena parcela de pacientes neutropênicos (10\%) no presente estudo se deve a eficácia do uso profilático do fluconazol nesta população, contrastando com a baixa utilização nos pacientes da unidade de terapia intensiva onde, não existe uma pratica de profilaxia apesar destes pacientes apresentarem muita condições associadas para ocorrência de candidemia. A ocorrência de Candida albicans (59\%) maior, portanto, que a tendência mostrada nos trabalhos dos últimos anos se deve neste estudo pela presença de $40 \%$ de crianças, onde predomina esta espécie. A predominância de Candida parapsilosis entre as crianças e de Candida glabrata entre os adultos, observado neste trabalho é similar a outros estudos ${ }^{102629}$. Candida parapsilosis é considerado agente de infecções exógenas por ser capaz de colonizar a pele, principalmente as mãos de profissionais da saúde, assim como as soluções glicosadas de uso hospitalar e cateter venoso central. Sua detecção é particularmente associada à nutrição parenteral total. A ocorrência de Candida parapsilosis é maior em crianças, principalmente em prematuros internados em unidades de terapia intensiva ${ }^{1032}$. Por outro lado, o motivo da predominância da Candida glabrata em adultos e principalmente idosos não é conhecido. Há um aumento na colonização bucal principalmente após os 60 anos, predominando a Candida albicans seguida pela Candida glabrata $^{19}$, podendo o mesmo ocorrer no restante do tubo digestivo, sendo necessários, portanto, estudos com este objetivo.

Destaca-se ainda a predominância de Candida tropicalis e Candida parapsilosis entre as espécies não albicans e a pequena ocorrência de Candida glabrata semelhante aos estudos brasileiros ${ }^{4311}$ contrastando com a maior ocorrência de Candida glabrata nos trabalhos americanos.

Entre as condições associadas as mais freqüentes foram uso de antibióticos (97\%), presença de cateter venoso central (77\%), bloqueador $\mathrm{H}_{2}$ (57\%), nutrição parenteral total (49\%), internamento na unidade de terapia intensiva (41\%), uso de corticosteróide (39\%), cirurgia do aparelho digestivo (35\%), similar a outros estudos ${ }^{142} 182024$. Observa-se o pequeno número de pacientes neutropênicos em virtude da eficácia da profilaxia com fluconazol nos pacientes da unidade de transplante de medula óssea, e neutropênicos devido à quimioterapia. A colonização é uma condição que precede a infecção e a incidência de apenas $33 \%$ reflete o fato de que culturas de vigilância não foram rotineiras durante o período de estudo. 0 bloqueador $\mathrm{H}_{2}$ não é um fator de risco direto de candidemia, e sim um marcador de gravidade da doença, pois é utilizado para prevenção de hemorragia digestiva alta em pacientes críticos. 
Candida glabrata e Candida krusei naturalmente apresentam suscetibilidade reduzida ao fluconazol. No entanto, tem-se observado taxas de resistência para Candida albicans, Candida tropicalis e Candida parapsilosis ${ }^{16152728}$. Entre os 51 isolados de Candida, cuja suscetibilidade a antifúngicos foi avaliada, três Candida glabrata apresentaram suscetibilidade dose-dependente ao fluconazol e resistência ao itraconazol, um isolado de Candida kruse $i$ apresentou suscetibilidade dose-dependente ao fluconazol, e um isolado de Candida pelliculosa suscetibilidade dosedependente ao itraconazol. Este perfil favorável de sensibilidade, também observado emoutros trabalhos brasileiros ${ }^{4311}$ deve-se provavelmente ao uso em pequena escala de fluconazol profilático, excetuando-se a unidade de transplante de medula óssea. Sessenta e oito pacientes receberam tratamento. Este dado é bastante importante uma vez que existe hoje um consenso que todos os pacientes com candidemia devem receber tratamento com antifúngicos. Nos 32\% de pacientes não tratados, a hemocultura positiva coincidiu com o dia do óbito e em alguns casos no post mortem. A taxa total de mortalidade foi de $56 \%$ apesar de $68 \%$ dos pacientes tratados mostrando que outras variáveis como gravidade da doença, idade, espécies de Candida, internamento na unidade de terapia intensiva influenciam no prognóstico.

0 presente estudo apresenta limitações devido ao fato dos pacientes de 2001-2002 pertencerem a série histórica e, portanto não foi possível análise se suscetibilidade aos antifúngicos neste grupo. Também não foi avaliado se o cateter venoso central foi mantido ou retirado após o diagnóstico de candidemia, sendo este um importante fator prognóstico. Finalmente, este estudo foi realizado somente em uma instituição pública, e o perfil favorável de suscetibilidade aos antifúngicos talvez não possa ser extrapolado para instituições privadas.

Este estudo mostra que candidemia piora o prognóstico de pacientes críticos. Destaca-se ainda uma incidência superior à observada em trabalhos internacionais. Embora não existam explicações bem definidas, acredita-se que a introdução de práticas simples de controle de infecção hospitalar, como a higienização das mãos, e possivelmente profilaxia antifúngica em pacientes de risco, o panorama possa modificar-se.

\section{AGRADECIMENTOS}

Agradecemos ao Prof. Arnaldo Lopes Colombo (Laboratório Especial de Micologia da Universidade Federal de São Paulo) e à equipe do Laboratório de Micologia do Hospital de Clínicas da Universidade Federal do Paraná.

\section{REFERÊNCIAS}

1. Abi-Said D, Anaisse E, Uzun O, Raad I, Pinzcoaski H, Vartivarian S. The Epidemiology of Hematogenous Candidiasis by Different Candida Species. Clinical Infectious Diseases 24: 1122-1128, 1997.

2. Alonso-Valle H, Acha 0, Garcia-Palomo JD, Farinas-Álvarez C, FernandezMazarrasa C, Farinas MC. Candidemia in Terciary Care Hospital: Epidemiology and Factors Influencing Motality. European Clinical Microbiology Infectious Diseases 22: 254-257, 2003.
3. Antunes AGV, Pasqualotto AC, Diaz MC, Azevedo PA, Severo LC. Candidemia ia a Brazilian Terctiary Care Hospital: Species Distribution and Antifungal Susceptibility Patterns. Revista do Instituto de Medicina Tropical de São Paulo 46: 239-241, 2004

4. Aquino VR, Lunardi WL, Goldmani LZ, Barth AL. Prevalence, Susceptibility Profile for Fluconazole and Risk Factors for Candidemia in a Tertiary Hospital in Southern Brazil. The Brazilian Journal of Infectious Diseases 9: 411-418, 2005.

5. Becke-Sague CM, Jarvis WR, and The National Nosocomial Infections Surveillance System. Secular Trends in The Epidemiology of Nosocomial Infections in The Unit States, 1980-1990. Journal Infectious Diseases 167: 1247-1251, 1993.

6. Blot SI, Vandewoude KH, Hoste EA, Colardyn FA. Effects of Nosocomial Candidemia on Outcomes of Critically ill Patients. The American Journal of Medicine 113: 480-485, 2002

7. Blumberg HM, Jarvis WR, Soucie JM, Edwards JE, Patterson JE, Pfaller MA, Rangel-Fraustom S, Rinaldi MG, Saiman L, WEiblin RT, Wenzel RP. Risk Factors for Candidal Blood Stream Infections in Surgical Intensive Care Unit Patients. The Nemis Prospective Multicenter Risk Factors for Candidal Bloodstrean Infections in Surgical intensive Care Unit Study. Clinical Infectious Diseases 33: 177, 2001.

8. Clark TA, Hajjeh HA. Recent Trends in The Epidemiology of Invasive Mycoses. Current Opinion in Infectious Diseases 15: 569-574, 2002.

9. Colombo AL. Epidemiology and Treatment of Hematogenous Candidiasis. A Brazilian Perspective. The Brazilian Journal of Infectious Diseases 4: 113-118, 2000 .

10. Colombo AL, Guimarães T. Epidemiology of Hematogenous Infections due to Candida spp. Revista da Sociedade Brasileira de Medicina Tropical 36: 599-607, 2003.

11. Colombo AL, Nucci M, Park BJ, Nouer SA, Arthington-Skaggs B, Matta DA, Warnock D, Morgan J, Brazilian Network Candidemia Study. Epidemiology of candidemia in Brazil: a nationwide sentinel surveillance of candidemia in eleven medical centers. Journal of Clinical Microbiology 44: 2816-2823, 2006.

12. Edmond MB, Wallace SE, McClish DK, Phaller MA, Jones RN, Wenzel RP. Nosocomial Bloodstream Infections in United States Hospitals: A Three-Year Analysis. Clinical Infectious Diseases 29: 239-244, 1999.

13. Eggimann P, Garbino J, Pittet D. Epidemiology of Candida Species Infections in Critically ill Non-imunosuppresed Patients. The Lancet Infectious Diseases 3: 685-702, 2003.

14. Hadley S, Lee WW, Ruthazer R, Nasraway SA. Candidemia as a Cause and Multiple Organ Failure in Non-imunocompromised Patients. Critical Care Medicine 30: 1808-1814, 2002.

15. Hajjeh RA, Sofair AN, Harrison LH, Lyon GM, Arthington-Skggs BA, Mirza SA, Phelan M, Morgan J, Lee-Yang W, Ciblak MA, Benjamin LE, Sanza LT, Huie S, Yeo SF, Brandt ME, Warnock DW. Incidence of Bloodstream Infectious Due to Candida Species and in vitro Susceptibilities of Isolates Collected from 1998-2000 in a Population-Based Active Surveillance Program. Journal of Clinical Microbiology 2: 1519-1527, 2004.

16. Hazen KC, Baron EJ, Colombo AL, Girmenia C, Sanches-Souza A, Palacio A, Bedout C, Gibbs DL. Comparison of Susceptibility of Candida spp. To Fluconazole and Voriconazole in a 4-Yara Global Evaluation Using Disk Diffusion. Journal of Clinical Microbiology 41: 5623-5632, 2003.

17. Jarvis WR. Epidemiology of Nosocomial Fungal Infections with Enphasis on Candida Species. Clinical Infectious Diseases 20: 1526-1530, 1995.

18. Karlowsky JA, Zhanel GG, Klym KA, Hoban DJ, Kabani AM. Candidemia in a Canadian Tertiary Care Hospital from 1976 to 1996. Diagnostic Microbiology Infectious Diseases 28: 5-9, 1997.

19. Lockart SR, Joly S, Vargas K, Swails-Wenger J, Enger L, Soll DR. Natural Defenses Against Colonization Breakdown in the Oral Cavities of the Elderly Journal of Dental Research 78: 857-868, 1999.

20. Luzzati R, Amalfitano G, Lazzarini L, Soldani F, Bellino S, Solbiati M, Danzi MC, Vento S, Todeschini G, Vivenza C, Concia E. Nosocomial Candidemia in NonNeutropenic Patients at an Italian Tertiary Care Hospital. European Journal Microbiology Infectious Diseases 19: 602-607, 2000.

21. Martin GS, Mannino DM, Eaton S, Moss M. The Epidemiology of Sepsis in The United States from 1979 through 2000. The New England Journal of Medicine 348: 1546-1554, 2003. 
22. Nguyen MH, Peacock LE, Morris AJ, Tanner DC, Nguyen ML, Snydman DR, Wagner MM, Rinaldi MG, Yu VL. The Changing Face of Candidemia: Emergence of NonCandida albicans Species and Antifungal Resistance. The American Journal of Medicine 100: 617-623, 1996.

23. Nucci M, Colombo AL. Risk Factors for Breakthrough Candidemia. European journal Microbiology Infectious Diseases 21: 209-211, 2002.

24. Nucci M, Silveira MI, Spector N, Silveira F, Velasco E, Akiti T, Barreiros G, Derossi A, Colombo AL, Pulcheri W. Risk Factors for Death Among Cancer Patients with Fungemia. Clinical Infectious Diseases 27: 107-111, 1998.

25. Pittet D, Li N, Woolson RF, Wenzel RP. Microbiological Factors Influencing of Nosocomial Bloodstream Infections: A 6-Year Validated, Population-Based Model Clinical Infectious Diseases 24: 1068-1078, 1997.

26. Pappas PG, Rex JH, Lee J, Hamill RJ, Larsen RA, Powderly W, Kauffman CA, Hyslop $\mathrm{N}$, Mangino JE, Chapman S, Horowitz HW, Edwards JE, Dismukes WE. For The NIAID Mycoses Study Group. A Prospective Obsrvational Study of Candidemia. Epidemiology, Therapy, and Influences on Mortality in Hospitalized Adult and Pediatric Patients. Clinical Infectious Diseases 37: 634-643, 2003.

27. Phaller MA, Diekma DJ, Messer SA, Boyken L, Hollis RJ. Activities of Fluconazole and Voriconazole against 1586 Recent Clinical Isolates of Candida Species Detrmined by Broth Microdilution, Disk Diffusion, and Etest Methods: Report from The ARTEMIS global Antifungal Susceptibility Program, 2001. Journal of Clinical Microbiology 41: 1440-1446, 2003.

28. Phaller MA, Messer SA, Hollis RJ, Jones RN, Diekma DJ. In vitro of Ravuconazole and Voriconazole Comoared with Those Of Four Approved Systemic Antifungal Agents against 6970 Clinical Isolates of Candida spp. Antimicrobial Agentes and Chemotherapy 46: 1723-1727, 2002

29. Pasqualotto AC, Nedel WL, Machado TS, Severo LC. A 9-year Study Comparing Risk Factors and the outcome of Pediatrics and Adults with Nosocomial Candidemia. Mycopathologia 160: 111-116, 2005.

30. Viscoli C, Girmenia C, Marinus A, Collette L, Martino P, Vandercam B, Doyen C, Lebeau B, Spence D, Kremery V, Pauw B, Meunier F, and the Invasive Fungal Group of the EORTC. Candidemia in Cancer Patients: A Prospective, Multicenter Surveillance Study by the Invasive Fungal Infection Group (IFIG) of the European Organization for Research and Treatmment of Cancer (EORTC). Clinical Infectious Diseases 28: 1071-1079, 1999.

31. Viudes A, Peman J, Canton E, Úbeda P, Lopez-Ribot JL, Gobernado M. Candidemia at a Terciary-Care Hospital: Epidemiology, Treatment, Clinical Outcome and Risk Factors for Death. European Journal of Microbiology Infectious Diseases 21:767-774, 2002.

32. Wingard JR. Importance of Candida species other than Candida albicans as pathogens in oncology patients. Clinical Infectious Diseases 20: 115-125, 1995. 